\title{
Proposal for further study of risk factors and health policy for human brucellosis in Northern Jordan
}

Sir,

Recently, we read with interest the article by Abo-Shehada and Abu-Halaweh [1] entitled "Risk factors for human brucellosis in northern Jordan." In 2004, the rate of human brucellosis in South Korea increased, and at that time, we conducted a case series investigation regarding the risk factors for the spread human brucellosis [2].

It is known that there is a high seroprevalance and incidence of human brucellosis in Jordan [3]. From your paper, we found that there are many cultural differences between Jordan and South Korea in the breeding of cattle and intake of beef and dairy products. For example, Koreans rarely consume unsterilized milk or dairy products, but often eat yukhoe, also known as Koreanstyle raw beef.

We were able to obtain a great deal of information through your paper. We are sending this letter to convey information regarding the management of brucellosis in South Korea as well as to ask some questions about your paper. In South Korea, brucellosis in cattle began to increase from 2002, and human brucellosis began to increase around the same time [4]. In the 2004 human brucellosis research, we were trying to determine other, more specific transmission routes, besides the known routes that exist. As a result, a major transmission route was identified related to cattle birth, where brucellosis patients had touched with their bare hands calves or placentas that were infected with the Brucella species.

On the basis of several epidemiologic investigation results, the Korea Center for Disease Control and Prevention advises all workers, particularly veterinarians and dairy farmers, to wear protective equipment, such as mask and gloves, when assisting cow births. The Korean government has also implemented a quarantine system and a thorough inspection of cattle brucellosis, as well as prevention activities among the high-risk groups. All cattle traded in markets and cattle older than 1 year must be tested for brucellosis [5]. Through these efforts, human and cattle brucellosis has been decreasing since 2007 and has now almost been eradicated in South Korea (Figure 1).

We have some questions for the above-mentioned paper. First, the authors used logistic regression for the multivariate analysis. But, in the matched case-control study, surely it is more appropriate to use a conditional logistic regression analysis. Second, we think there are many transmission routes related to cattle birth in Jordan. Therefore in the study were detailed question asked, such as "Have you ever handled an abortus or a premature calf with bare hands?". If the questions about "contact" were in detail, useful information may have been missed. Thus in-depth questioning may help brucellosis management.

\section{References}

1. Abo-Shehada MN, Abu-Halaweh M. Risk factors for human brucellosis in northern Jordan. Eastern Mediterranean Health Journal, 2013, 19(2):135-140.

2. Lim HS, Min YS, Lee HS. [Investigation of a series of brucellosis cases in gyeongsangbukdo during 2003-2004]. Journal of Preventive Medicine and Public Health, 2005, 38(4):482-488 [in Korean].

3. Abo-Shehada MN et al. Seroprevalence of brucellosis among high risk people in northern Jordan. International Journal of Epidemiology, 1996, 25(2):450-454.

Young-Sun Min

Department of Preventive Medicine, College of Medicine, Dongguk University, Gyeongju, South Korea (mys0303@gmail.com).

Hyun-Sul Lim

Department of Preventive Medicine, College of Medicine, Dongguk University, Gyeongju, South Korea.

The authors of "Risk factors for human brucellosis in northern Jordan" [1] were invited to respond to this letter, but no response was received.
1. Korea Center for Disease Control and Prevention. [Current situation of human brucellosis]. CDMR, 2006, 17(2):1-7 [in Korean].

2. National Veterinary Research and Quarantine Service. [News of primary project]. Vet Res Quarantine Inf , 2006, 32:26-30 [in Korean]. 


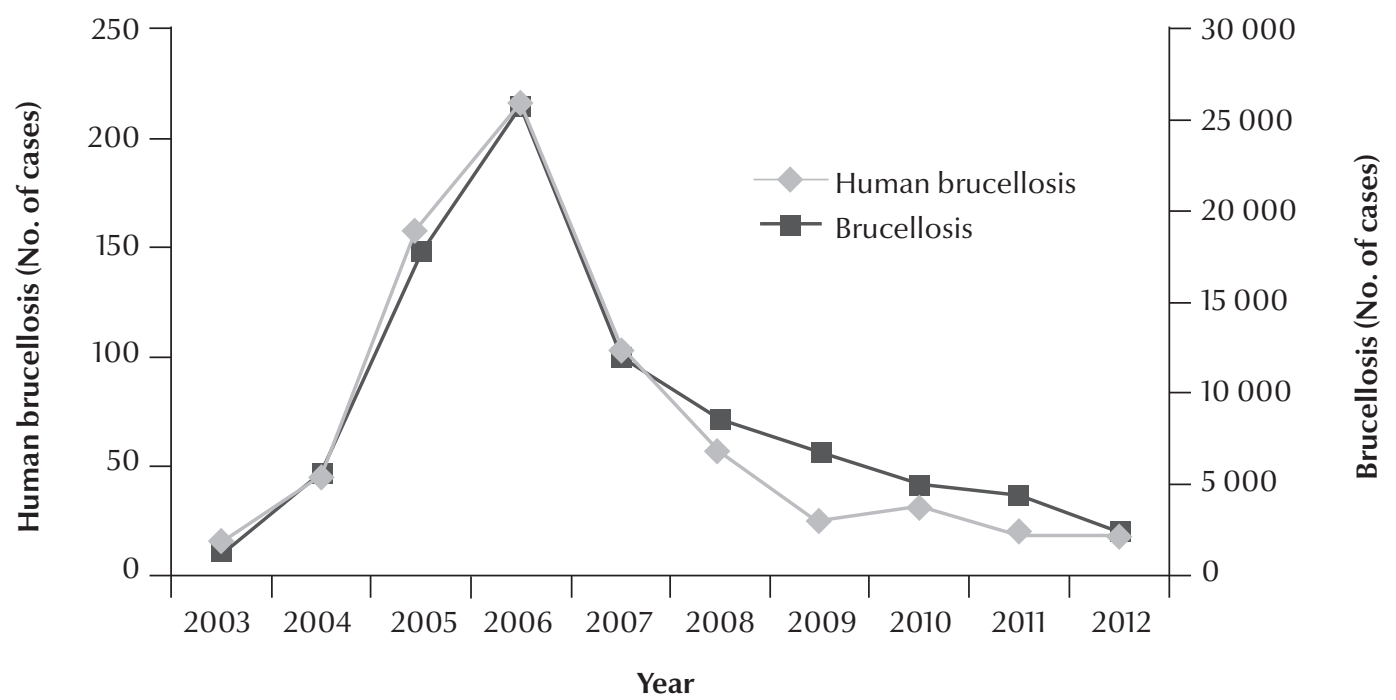

